\title{
https://doi.org/10.1016/j.jenvman.2021.113359
}

This manuscript has been ACCEPTED for publication in the JOURNAL OF ENVIRONMENTAL MANAGEMENT. This manuscript has been peer reviewed.

When available, the final version of this manuscript will accessible via the 'Peer-reviewed Publication DOI' link on the right-hand side of this webpage. Please feel free to contact any of the authors; we welcome feedback! 


\section{A machine learning approach for prioritizing groundwater testing for per-and \\ 2 polyfluoroalkyl substances (PFAS)}

3 Sarabeth George ${ }^{\mathrm{a}, *}$, Atray Dixit ${ }^{\mathrm{b}}$

$4 \quad{ }^{a}$ California State Water Resources Control Board; sarabethgeorge@gmail.com

5 bCoral Genomics, San Francisco, CA 94107, USA; atray@coralgenomics.com

$6 \quad *$ Corresponding author

8 Abstract

9 Regulatory agencies are beginning to recognize and regulate per-and polyfluoroalkyl substances

10 (PFAS) as concerning environmental contaminants. In groundwater management, testing and

11 mitigation strategies are desirable, but can be time and cost-intensive processes. As a result, only

12 a fraction of all groundwater wells has been tested for PFAS levels, resulting in potentially

13 extended drinking water exposure to PFAS in the meantime. In this study, we build a series of

14 machine learning models (including linear and random forest regressors) to predict PFAS based

15 on a groundwater dataset from California. These models are used to compare the relative predictive

16 ability of co-contaminant fingerprints, hydrological properties, soil parameters, proximity of

17 airports/military bases, and geospatial data. Additionally, a random forest machine learning model

18 that combines all data types can quantitatively predict the maximum PFAS compound

19 concentration in a well with a Spearman correlation of 0.64 and can discern wells containing

20 concerningly high concentrations of PFAS with an accuracy of $91 \%$ (AUC of 0.90 ). This approach

21 may have widespread utility for other hazardous anthropogenic compounds in groundwater. Future

22 investigations should evaluate the practicability of using machine learning to prospectively

23 prioritize contaminant testing in groundwater wells. 


\section{Introduction:}

25 Per-and poly- fluoroalkyl substances (PFAS) are a class of water-soluble anthropogenic

26 contaminants of increasing global concern due to their ubiquity in the global environment,

27 persistence, and toxicity. Management of PFAS contamination in water resources requires cross-

28 cutting approaches in multiple industries, from management of solid and liquid wastes to

29 monitoring and regulation of industrial operations and drinking water. PFAS have been used

30 extensively in many products including textile coatings, surfactants, pesticides, food contact

31 materials, and fire-fighting foams since the mid-20 $0^{\text {th }}$ century (Prevedouros et al., 2006; Wang et

32 al., 2017). The primary source of PFAS to the environment is estimated to be industrial emissions

33 (Prevedouros et al., 2006), however, PFAS are also known to be formed as the result of breakdown

34 of "precursor" compounds such as fluorotelomer alcohols and perfluoroalkyl sulfonamido alcohols

35 (Fasano et al., 2006; Martin et al., 2006, 2005). Many studies have shown that PFAS compounds

36 persist in the environment, bioaccumulate, and are toxic (Conder et al., 2008; De Silva et al., 2021;

37 Frömel and Knepper, 2010; Houtz et al., 2013; Parsons et al., 2008; Young and Mabury, 2010).

38 PFAS have been detected throughout the global environment, biota, and humans; the 2011-2012

39 United States National Health and Nutrition Examination Survey found detectable PFAS in 97\%

40 of individuals tested (Giesy and Kannan, 2001; Lewis et al., 2015; Rayne and Forest, 2009;

41 Vestergren and Cousins, 2009). Studies have linked several PFAS, including perfluorooctanonate

42 (PFOA) and perfluorooctane sulfonate (PFOS), with adverse effects on environmental and human

43 health, including decreased birth weight and increased incidence of liver, pancreas and testicular

44 tumors (Biege et al., 2001). The primary pathways of human exposure to PFAS is include diet,

45 indoor environments polluted with PFAS, and PFAS-contaminated drinking water (Domingo and

46 Nadal, 2019; Sjogren et al., 2016; Vestergren and Cousins, 2009). Blood serum PFAS in humans 
47 has been shown to be positively related to drinking water PFAS concentration (Ericson et al., 2008;

48 Kannan et al., 2004; Xu et al., 2020).

49 In California, the geographic focus of this study, approximately $40 \%$ of drinking water is supplied

50 by groundwater wells. In drought years, up to $60 \%$ of drinking water is sourced from groundwater

51 (Carle, 2015). PFAS has been detected in California in both the influent and effluent of wastewater

52 treatment plants (Houtz et al., 2018); in urban stormwater runoff (Houtz and Sedlak, 2012; Plumlee

53 et al., 2008); in urban rivers (Plumlee et al., 2011; Sengupta et al., 2014); and in the tissues of

54 marine mussels (Dodder et al., 2014). Between 2013-2015, six PFAS contaminants were

55 systematically sampled in drinking water in the United States for the first time as part of the United

56 States Environmental Protection Agency (USEPA) Third Unregulated Monitoring Rule, or

57 UCMR3 Report; this study showed widespread PFAS contamination at concerning concentrations

58 in water samples nationwide, including detections in California (Crone et al., 2019; Hu et al., 2016;

59 US EPA, 2015). Furthermore, recent studies have found significant correlations between drinking

60 water PFAS concentration and sera concentrations of PFAS in Northern California (Hurley et al.,

61 2016; Kim et al., 2020). In response to growing concerns and public pressure regarding PFAS

62 contamination in California, the California State Water Resources Control Board (State Water

63 Board) began issuing investigative orders in 2019 , targeting high-risk drinking water systems and

64 known potential source sites (State Water Resources Control Board, 2020). Results of these

65 analyses have been publicly shared on the Groundwater Ambient Monitoring and Assessment

66 (GAMA) database.

67 Both in the State of California, and globally, current frameworks for prioritizing drinking water

68 testing for PFAS are poorly suited to identifying contamination in areas not directly adjacent to

69 known source industries and facilities; furthermore, representative sampling of the more than one 
70 million groundwater wells in California, particularly private wells, will take years. This study

71 presents a novel method for risk assessment of PFAS contamination using co-contaminant

72 fingerprints, airport and military installation proximity, hydrological, soil and geospatial data as

73 features to train a machine learning model. The hypotheses driving this investigation are (1) that

74 anthropogenic contaminants, including PFAS, follow consistent patterns in the environment both

75 in terms of patterns of release from source areas and transport/transformation over time (Alimi et

76 al., 2003; Barrett et al., 1999; Douglas et al., 2007; Kibbey et al., 2020; Le et al., 2021) and (2)

77 these consistent patterns can be leveraged to predict the risk that PFAS will exceed regulatory

78 thresholds using supervised machine learning. In the future, predictions from supervised machine

79 learning applications could be used to prioritize sampling of the highest-risk drinking water wells,

80 reducing human exposure to unsafe concentrations of PFAS.

\section{Materials and Methods}

\section{Generation and curation of dataset}

83 Data was downloaded from the Geotracker Groundwater Ambient Monitoring and Assessment

84 (GAMA) database (California State Water Resources Control Board, 2020). The GAMA

85 Program is California's comprehensive groundwater quality monitoring program that was created

86 by the State Water Board in 2000. It was later expanded by Assembly Bill 599-the Groundwater

87 Quality Monitoring Act of 2001-requiring the State Water Board to assess groundwater quality

88 in basins that account for $95 \%$ of the state's groundwater use. This system provides access to

89 approximately 87 million analytical results from over 290,000 wells in California for more than

90200 analytes, spanning the 1960 's to present. Notably, given the fact that PFAS in groundwater

91 has been linked use of fire-fighting foams at military bases in the United States, military 
92 installation groundwater monitoring data is generally not available in GAMA, including recent

93 US Department of Defense studies on PFAS (Hu et al., 2016).

94 For this study, the dataset was filtered based on duplicate wells and wells that had fewer than 5

95 analyte measurements to a set of 189,972 wells across 228 analytes (approximately $4.7 \%$ of the

96 values of this dataset are not missing); for wells where analyte data was available for multiple

97 sampling events, the average value was used. The chemical data for each well was normalized

98 using a $\log 10($ analyte in parts per trillion $(\mathrm{ppt})+1)$ transformation. It was then combined with

99 geospatial data including latitude, longitude, and other metadata. The summary statistics for the

100 maximum PFAS concentration for each well is summarized in Table $\mathbf{1}$ below and for the broader

101 set of co-contaminants in Table S1.

102 Other datasets included: the number of nearby (within 1.5 mile radius) airports/military bases,

103 depth to groundwater, elevation, 15 soil hydraulic properties, and 15 features representing other

104 soil properties. These data types (and their sources) are summarized in Table 2.

105 Table 1: Summary of PFAS measurements in dataset. Values are based on a $\log _{10}$ (PFAS

106 compound in ppt+1) scale.

\begin{tabular}{|l|l|l|l|l|l|}
\hline & \# of wells & mean & std & min & max \\
\hline $\begin{array}{l}\text { Maximum } \\
\text { PFAS value } \\
\text { per well }\end{array}$ & 1410 & 1.30 & 0.77 & 0.01 & 5.0 \\
\hline
\end{tabular}

108 Table 2: Summary of the various feature categories/subsets used in the machine learning model 109 to predict PFAS. 


\begin{tabular}{|c|c|c|c|}
\hline $\begin{array}{l}\text { Feature } \\
\text { (name used in } \\
\text { paper, if } \\
\text { different) } \\
\end{array}$ & $\begin{array}{l}\text { Number } \\
\text { of } \\
\text { features }\end{array}$ & Description & Source \\
\hline $\begin{array}{l}\text { Nearby airports } \\
\text { and military } \\
\text { bases } \\
\text { (airport/military } \\
\text { base proximity) }\end{array}$ & 9 & $\begin{array}{l}\text { The number of nearby large, medium, } \\
\text { or small airports, seaplane bases, } \\
\text { heliports and whether they are active } \\
\text { or closed. } \\
\text { AND } \\
\text { The number of nearby large, medium, } \\
\text { or small military bases and whether } \\
\text { they are active or closed. }\end{array}$ & 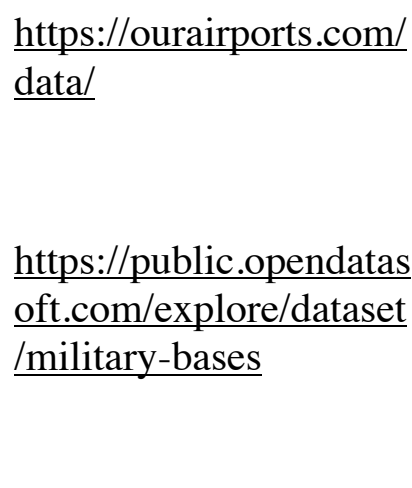 \\
\hline Geospatial & 3 & Latitude, longitude, and elevation & $\begin{array}{l}\text { (“Elevation Point } \\
\text { Query Service,” n.d.; } \\
\text { State Water Resources } \\
\text { Control Board, 2020) }\end{array}$ \\
\hline $\begin{array}{l}\text { Hydrology and } \\
\text { soil hydraulic } \\
\text { properties } \\
\text { (Hydrology) }\end{array}$ & 15 & $\begin{array}{l}\text { Depth to groundwater } \\
\text { Means and standard deviations } \\
\text { associated with field capacity, } \mathrm{h}_{\mathrm{m}}, \mathrm{K}_{\mathrm{s}} \text {, } \\
\text { Plant available water, Sigma, } \theta_{\mathrm{r}}, \theta_{\mathrm{s}}\end{array}$ & $\begin{array}{l}\text { California Natural } \\
\text { Resources Agency, n.d. } \\
\text { and Zhang et al., } 2018\end{array}$ \\
\hline Soil & 15 & Terrain, land cover, soil quality & Fischer et al., 2008 \\
\hline $\begin{array}{l}\text { Co- } \\
\text { contaminants }\end{array}$ & 228 & $\begin{array}{l}\text { Groundwater quality data for over } \\
290,000 \text { wells in California }\end{array}$ & $\begin{array}{l}\text { (State Water Resources } \\
\text { Control Board, 2020) }\end{array}$ \\
\hline
\end{tabular}

$111 \quad$ Linear Model

112 Linear regression is a simple machine learning method that can be used to solve for the best fit

113 line relating each of a set of features to a target variable of interest. In this paper we use it as a

114 benchmark to compare performance against nonlinear models (such as Random Forest

115 Regression, described below). We use the sklearn.linear_model.Ridge method from Python 
116 which applies a penalty term for slopes (coefficients) that have a large magnitude in order to

117 reduce the likelihood of overfitting.

118 Random Forest Regression

119 Random forest regression is an ensemble method that uses a collection of decision trees to derive 120 estimations of the likely value of a target variable given a set of features. When using a Random

121 Forest Regressor, the sklearn.ensemble.RandomForestRegressor method in Python was used.

122 The number of estimators was set to 1000 and the min_samples_leaf was set to 2 . The

123 performance of the Random Forest Regressor was evaluated based on cross-validation described 124 below.

125 Cross-validation: random and geospatially localized grouping

126 In order to evaluate the performance of our machine learning approach to predict PFAS levels, 127 wells were grouped into 10 equal sized subcomponents. We created these groups using two 128 separate methods. In the first, groundwater wells were randomly assigned to each of 10 groups

129 (map in Figure 3A). In the second, data was split using a modified version of k-nearest

130 neighbors clustering such that each group contained wells from a unique geospatial region.

131 (Figure 3B).

132 For each of these approaches, cross-validation was performed with nine of the groups used for

133 training the machine learning model and the last group used as a test set to evaluate the model's

134 performance (on data the model had not been trained on). This cross validation was repeated

135 until each group had been used as the test set.

137 Step by step procedure of model training, evaluation, and application 
1. Collate the feature subsets (airport/military base proximity, geospatial data, soil properties, hydrologic properties, co-contaminants), and a combined feature set with all of the subsets for all wells with the analyte of interest (in the case of PFAS, 1,410 wells).

2. Mask the analyte of interest from feature sets containing co-contaminant data (in the case of predicting the maximum PFAS value, all chemicals in the PFAS class were masked).

3. Divide the data into 10 groups using geospatial clustering or geospatially random cross validation approach described in Cross-validation above.

4. Train separate linear model and random forest regressors (as described above) to predict the analyte of interest trained on nine out of the 10 groups using each of the feature subset as well as the combined feature set.

\section{Capture the predicted value on the hold out group as well as the Spearman correlation} between the actual and predicted value.
6. Repeat across all groups.

7. Determine the best performing model.

8. Apply best-performing model to predict analyte levels of wells that have not been tested.

\section{Derivation of confidence interval estimates from Random Forest Regressor}

154 In order to create estimates of the robustness of the machine learning model predictions, we 155 leveraged the bagged nature of the Random Forest model (in which the estimates associated with 156 many decision trees are averaged) to derive an estimate of the variance in the prediction across 157 all trees (Wager et al., 2014). This method is implemented in the forestsci package in python. 
160 The plotly express package in python was used to plot and visualize PFAS values on a map of

161 California.

162 Results:

163 We sought to leverage the public datasets available through the California GAMA system as

164 described in Methods, to build a machine learning approach that could predict groundwater

165 wells with the greatest risk for high PFAS concentration (Figure 1). The machine learning model

166 predicts known PFAS measurements given other chemical measurements (i.e. potential co-

167 contaminants of PFAS), airports/military base proximity, depth to groundwater, soil hydraulic

168 properties, soil properties, (Supplementary Table 1) and geospatial data (elevation, latitude, and

169 longitude). This machine learning model can be applied to wells in which PFAS levels have not

170 been measured in order to predict PFAS concentration at that location (Figure 1C, D).

\section{A machine learning approach for prioritizing groundwater testing for per-and polyfluoroalkyl substances (PFAS)}

A

B

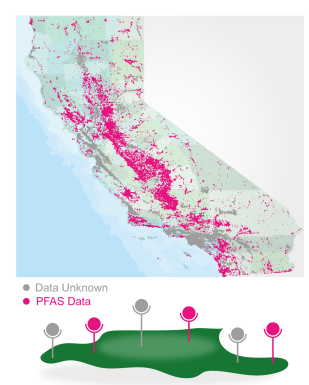

C

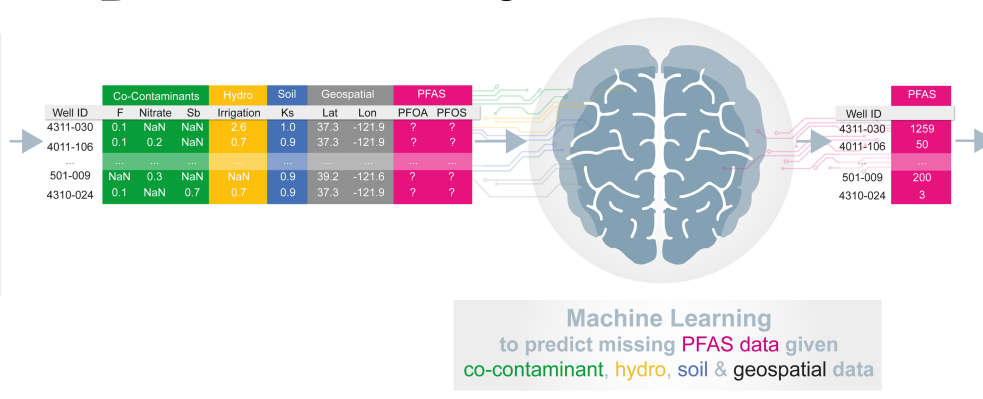

D

Unknown PFAS levels are
predicted using the ML mode

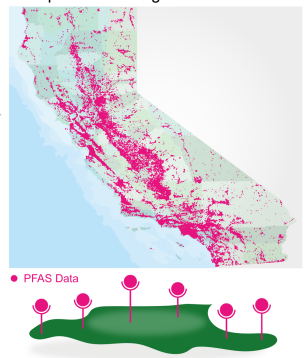

Figure 1. An overview of a machine learning approach to enable proactive water resource management for PFAS

174 (a) Visualization of the location of each of the 189,972 groundwater wells in the State of 
data types (green), hydrological data (yellow), soil data (blue), geospatial data (gray), and PFAS

177 measurements (pink) used in this study; airport/military base proximity data is not included in

178 this figure for simplicity. Columns correspond to different measurements and rows correspond to

179 different wells. "NaN" indicates to missing feature data and question mark symbols indicate

180 missing PFAS data that the machine learning model seeks to predict. (c) A machine learning

181 approach is used to predict PFAS data (pink) given the other data types. (d) This machine

182 learning model can then be applied to predict unknown PFAS levels in wells with no PFAS

183 testing performed, which can be in turn used to prioritize testing.

184 A diverse set of features used as inputs to a machine learning model trained on existing PFAS 185 data

186 PFAS measurements in our curated dataset were distributed throughout the State of California

187 (Figure 2A). We sought to incorporate diverse data types that may potentially be predictive of

188 PFAS levels (see Table 2, Figure 2B, C). A particular challenge in this co-contaminant data,

189 and in other similar monitoring datasets, is that very few wells have been tested across every

190 possible analyte. In the entire dataset, only $4.7 \%$ of chemical measurements across all possible

191 analytes and all possible wells have been tested. A naïve solution to handling this level of

192 missing data would be to focus on a subset of analytes and wells in which the data is relatively

193 complete. However, this would discard a tremendous resource associated with rich co-

194 contaminant correlation structure in this dataset (Figure 2C and Supplementary Figure S1).

195 There are many non-PFAS analytes/features with strong associations with PFAS compounds

196 such as a strong positive relationship between TBA (Tertiary butyl alcohol) and PFOA and a

197 weak negative relationship between PFUNDCA (perfluoroundecanoic acid) and SC (specific 

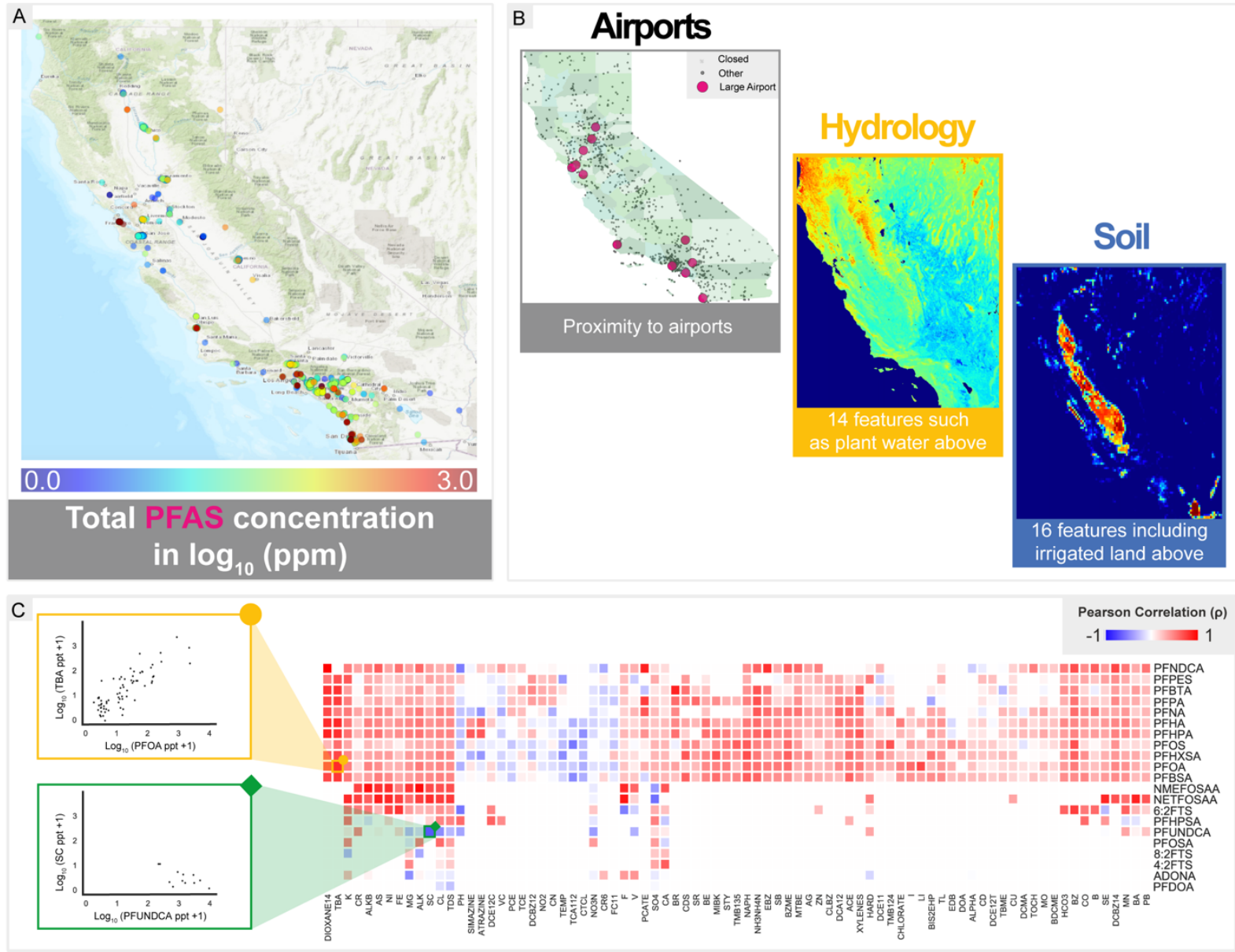

201 Figure 2. A diverse set of features used as inputs to a machine learning model trained on 202 existing PFAS data.

203 (a) Visualization of the location of each of the 1,410 groundwater wells (dots) in the State of

204 California that had associated PFAS measurements in our dataset. Each well is colored by total

205 PFAS concentration on a $\log 10$ scale. (b) Geospatial visualization of different feature types from

206 left to right: airports in California with larger pink dots corresponding to large airports, hydrology

207 and hydraulic soil properties, and soil properties. (c) A correlation matrix visualization of pairwise 
209 Pearson correlation between the respective elements as shown in the yellow and green scatter plots.

210 The color scale ranges from red to blue, corresponding to a correlation between 1.0 and -1.0.

211 Different machine learning models to evaluate importance of feature subsets and nonlinearities

212 We created several machine learning models to predict the maximum PFAS value for a given

213 well. Separate models were developed using each of the feature categories in order to provide an

214 estimate of their relative predictive power as well as a combined model across all features. For

215 each of these cases, we evaluated the performance of a regularized linear regression in

216 comparison to a random forest model using two forms of cross validation (see Methods). By

217 comparing the results from a cross validation in which wells were randomly grouped, to a cross

218 validation performed on wells geospatially separated from wells used for training, we were able

219 to understand the extent to which the model's predictions are highly dependent on local regional

220 variation. By comparing the results from a linear model to those of a random forest, we were

221 able to understand the relative importance of nonlinear interactions between features for the

222 prediction task.

223 Machine learning model accurately predicts individual PFAS compounds and overall PFAS

224 abundances

225 We find that for cross-validation in which wells are geospatially randomized, each feature subset 226 can be used independently to predict PFAS. Spearman correlations between predicted and actual 227 maximum PFAS value ranged from 0.19 for the airport proximity model $\left(\mathrm{p}<10^{-4}\right)$ to 0.64 for the 228 combined model $\left(\mathrm{p}<10^{-9}\right)$. Additionally, for all feature subsets except the number of nearby 229 airports, a random forest regression significantly outperformed the linear model (p-values 0.58 , 
231 contaminants respectively). Finally, a combined model across all features outperformed any of

232 the individual feature subsets (Figure 3A). We demonstrate strong correlation of the predictions

233 of this combined model with the actual measured maximum PFAS level along with uncertainty

234 estimates in the model predictions (Figure 3C). For cross-validation in which wells are

235 geospatially clustered, co-contaminant features were the most reliably predictive $\left(\mathrm{p}<5 \times 10^{-5}\right)$. A

236 combined model across all features did not outperform the co-contaminant alone model $(\mathrm{p}=0.98)$.

\section{7 (Figure 3B)}

238 We then applied the results of our ML approach to all wells in the GAMA dataset that have not

239 yet been tested. This resulted in a nearly 130 -fold increase in the number of wells with PFAS

240 estimates as well as the identification of a sizable number of wells with concerningly high

241 predicted PFAS levels (Figure 3D). We assessed the extent to which wells where PFAS has been

242 measured (our training / testing data) differ significantly from wells that have not been measured

243 for PFAS with respect to their co-contaminant profile. While the wells do not differ drastically

244 from other wells with respect to co-contaminant profiles (see dimensionality reduction

245 visualization in Figure S3A), we note that wells that have had PFAS levels measured have

246 significantly had more co-contaminant measurements performed on them than wells that have

247 not had PFAS measured (Figure S3B). 

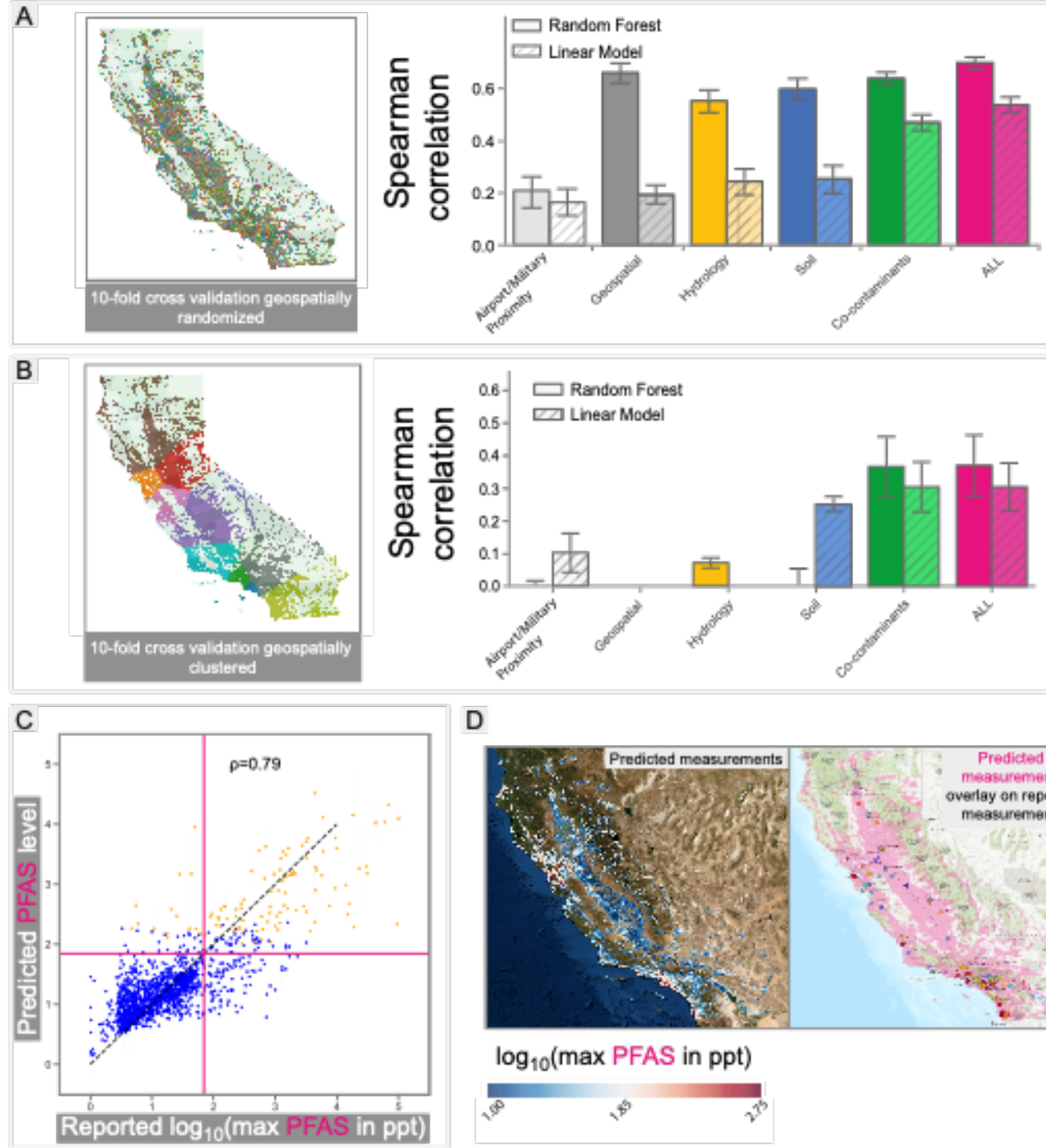

D
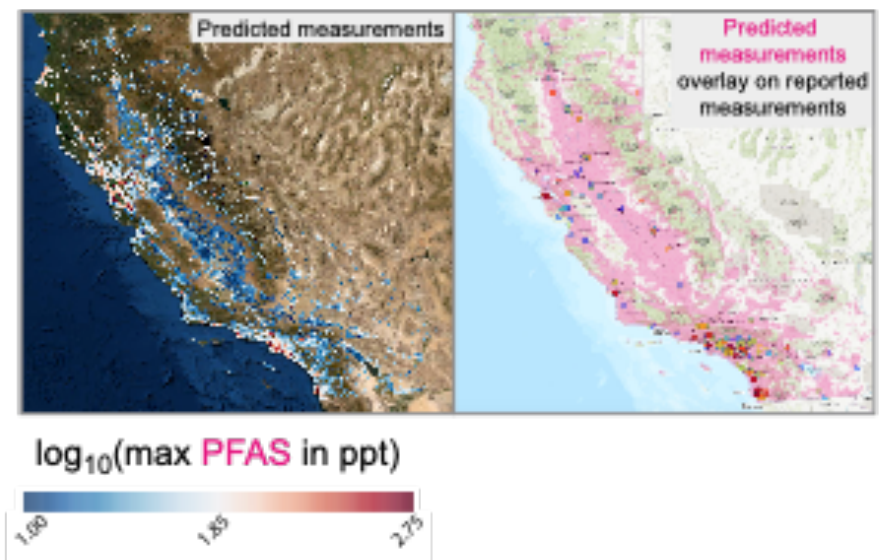

250 Figure 3. Machine learning model accurately predicts individual PFAS compounds and 251 overall PFAS abundances

252 (a) Results from models evaluated using a geospatially randomized cross validation. Wells were 253 assigned randomly to one of 10 categories as shown on the map on the left (colors correspond to 
254 various categories). Bar plots for model performance across each of the 10-folds on right for each

255 of the feature subsets and combined model. Y-axis corresponds to Spearman correlation between

256 predicted and actual maximum PFAS level ( $\left.\log _{10} \mathrm{ppt}\right)$ across wells in test set. Error bars represent

$25795 \%$ confidence intervals. (b) Results from models evaluated using a geospatially clustered cross

258 validation. Wells were assigned to one of 10 categories based on geospatial locations as shown on

259 the map on the left (colors correspond to various categories). Bar plots for model performance

260 across each of the 10-folds on right for each of the feature subsets and combined model. Y-axis

261 corresponds to Spearman correlation between predicted and actual maximum PFAS level $\left(\log _{10}\right.$

262 ppt) across wells in test set. Error bars represent 95\% confidence intervals. (c) Scatter plot between

263 reported maximum PFAS level (x-axis, $\log _{10} \mathrm{ppt}$ ) and predicted maximum PFAS level (y-axis).

264 Error bars correspond to two standard deviations of model predictions. Horizontal and vertical

265 lines correspond to $70 \mathrm{ppt}$. Orange dots correspond to wells whose lower bound on predicted

266 maximum PFAS level is above 70 ppt. (d) Map of predicted PFAS levels across all 189,972

267 groundwater wells.

268 Practical advantage of a risk adjusted testing strategy based on a machine learning model.

269 Lastly, we sought to quantify the model's performance when applied to a specific regulatory

270 threshold. We quantified its performance when identifying wells that have a maximum PFAS

271 concentration greater than $70 \mathrm{ppt}$, equivalent to the current USEPA Health Advisory Level for

272 the sum of PFOA and PFOS. The $70 \mathrm{ppt}$ threshold was chosen as a benchmark measure for wells

273 that may present elevated risk, although our analysis includes additional PFAS besides PFOA

274 and PFAS. At a 5\% false positive rate, the model recovered $67 \%$ of the true positive results in an

275 overall area under the curve of 0.90 (Figure 4A). To demonstrate how this model would be used

276 in a real world context, we simulated how quickly all wells with maximum PFAS levels greater 
277 than 70 ppt would be discovered using various strategies. We compare a strategy in which the

278 wells with the highest machine learning predicted PFAS levels were tested first with either a

279 random guessing strategy or a strategy in which the wells are guessed perfectly (Figure 4B). The

280 machine learning-informed strategy significantly outperformed a random sampling approach

$281 \quad\left(\mathrm{p}<10^{-7}\right)$

282

A

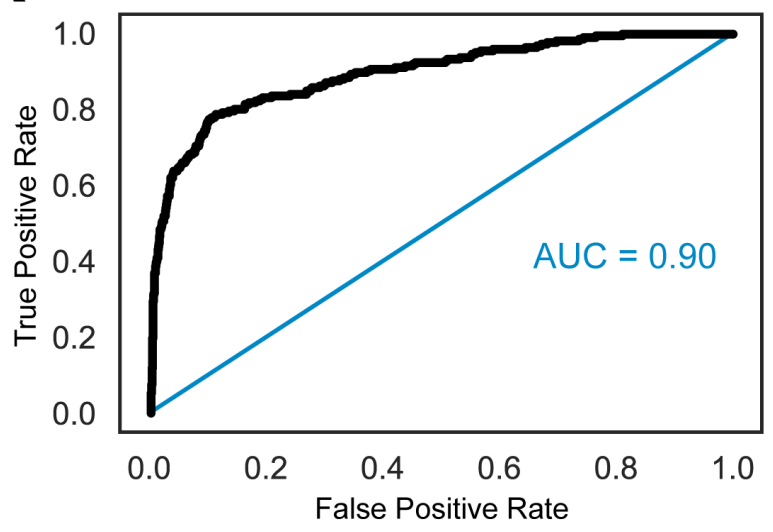

B

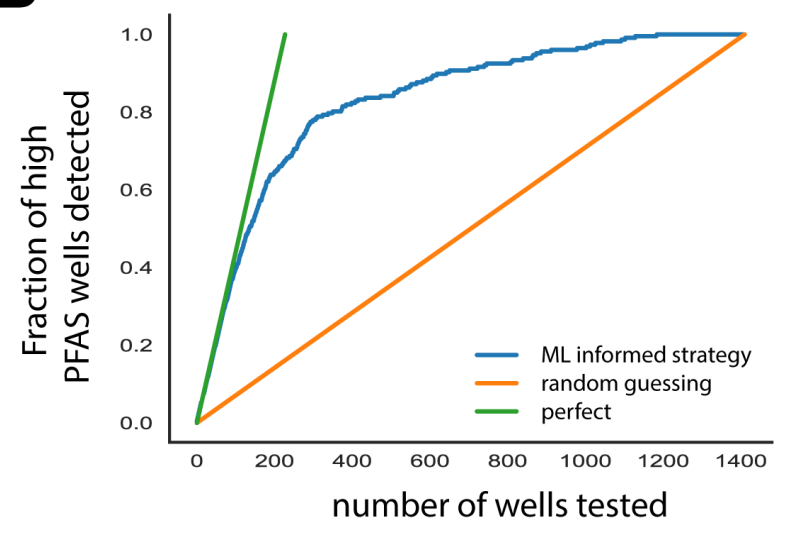

285 model.

(a) Receiver operating characteristic curve demonstrating model performance for predicting wells

287 where the maximum PFAS exceeds 70 ppt. Blue diagonal line represents the expected model

288 performance for a model which guesses randomly based on the average likelihood of a well

289 exceeding the threshold.(b) Results of a machine learning-informed testing strategy in which wells

290 with a predicted maximum PFAS level greater than 70 ppt are tested first (blue line). This result

291 is compared to a strategy in which wells are tested randomly (orange line) and one in which wells

292 with maximum PFAS level greater than 70 ppt are predicted perfectly (green line). 


\section{Discussion:}

296 Co-occurrence of chemical analytes in drinking water can be used for accurate machine

297 learning approaches for PFAS

298 A machine learning model based on co-contaminant data alone was able to significantly predict

299 maximum groundwater PFAS concentration regardless of whether or not it was trained on wells

300 in a similar geographic area to those upon which its performance was evaluated. Co-contaminant

301 data may act as a proxy for water management practices like water recycling and wastewater

302 injection in features such as ions $\left(\mathrm{Ca}^{2+}, \mathrm{Mg}^{2+}, \mathrm{Na}^{+}\right)$and parameters including specific

303 conductivity. Land use practices are likely also reflected in co-contaminant data: various regional

304 industries drive requirements to monitor groundwater for different contaminants regionally (e.g.,

305 pesticides in agricultural areas and chlorinated solvents in industrial areas). The addition of

306 airport/military base proximity, geospatial data, soil properties, and hydrologic properties boost

307 model performance, but only when the wells used for evaluating performance geographically

308 overlap with those used for training the model.

\section{Generalization across other contaminants and geographies}

310 Our results indicate that predictions using co-contaminant features are more generalizable across

311 contaminants and geographies than predictions using more locally specific features such as

312 geospatial, hydrologic and soil parameters. In geospatially randomized cross-validation trials,

313 each feature set (co-contaminants of PFAS, nearby airports, soil hydraulic properties and

314 geospatial data) can be used independently to predict PFAS, however, in geospatially clustered 
315 cross-validation trials, predictive power across all features was reduced. This pattern is generally

316 conserved across the 228 analytes in our dataset, notably, for many analytes for which the total

317 number of measurements exceeds that available for PFAS by $>10$ fold, e.g. 1,1-Dichloroethene

318 (DCE11) (Figure S3B). Overall, models to predict PFAS perform best when trained on wells in

319 geographically similar areas to those it is ultimately used to predict unknown values.

321 Recommendations to stakeholders

322 In the United States, State governments are beginning to make historical groundwater (and other

323 environmental) monitoring data available to the public, but the potential of these data resources

324 has thus far been underappreciated. Generally, the performance of machine learning methods

325 such as those outlined in this paper improve with the amount of data available; indeed, we

326 observed significant correlation between the size of the training dataset and model accuracy

327 when we applied our integrated machine learning model approach to each of the 228 analytes in

328 the dataset, $\mathrm{p}<10^{-9}$, Figure S3). We have demonstrated the potential of aggregating groundwater

329 contaminant measurements despite a significant level of sparsity with a high degree of missing

330 data elements (in this case excess of $>95 \%$ missing). This result highlights the need for

331 collaborations between regulatory bodies across state and national boundaries to bring together

332 environmental datasets in an open-source data framework. In such a framework, machine

333 learning methods such as these may be able to more rapidly identify and mitigate the most

334 dangerous sources of anthropogenic groundwater contamination. In order for the GAMA dataset,

335 and other similar public datasets to reach their full potential to support protection of human

336 health and the environment, cooperation between both regional and Federal governments is

337 imperative. For example, in the GAMA dataset, a significant shortcoming is that results from 
338 military bases are generally absent, because results from Department of Defense (DOD) cleanup

339 projects are not uploaded to the GAMA database; since DOD installations are a known source of

340 PFAS, this is a significant blind spot.

341 Application of computational techniques on existing monitoring datasets is a promising method

342 for improving detection of groundwater wells which pose a threat to human health. This

343 technique may even reveal previously unknown areas of concern for well-characterized

344 contaminants in addition to its utility in investigating emerging contaminants like PFAS. As

345 more regional and Federal governments make these data available, it will become possible to

346 create massive datasets of high-resolution historical groundwater data for many contaminants,

347 which may aid in scientific understanding of patterns of release and transport. Better

348 understanding of release and transport of contaminants that pose a threat to public health will

349 support proactive management and protection of groundwater resources, both by enabling

350 regulation of facilities and industries found to discharge contaminants of concern, and by

351 optimizing pumping, treatment and blending of extracted groundwater used as drinking water to

352 protect human health.

353 Machine learning tools such as those described in this study have the potential to aid in better

354 mitigation of the cumulative environmental contamination burden in high-risk communities by

355 providing robust integrated predictions of exposure to contaminant classes such as PFAS. We

356 recommend that these tools be used to implement additional testing of the highest-predicted-risk

357 wells rather than as a method for determining entire monitoring schedules. This is because with

358 the application of any computational method that makes predictions on historical data, inherent

359 biases in the data (e.g., exclusion of DOD data) are a significant concern when evaluating and

360 applying predictions, especially when predictions can affect public health. In particular, we 
361 emphasize the potential environmental justice pitfalls of relying naïvely on machine learning

362 predictions to make regulatory decisions. In the United States, heavy-polluting industries and

363 facilities have historically been built in or adjacent to socioeconomically and racially segregated

364 neighborhoods due to a combined history of exclusionary residential real estate practices and

365 zoning laws (Maantay, 2002; Mohai et al., 2009; Mohai and Saha, 2015). These invisible

366 sociohistorical overprints on the groundwater monitoring data are not unique to the United States

367 and will need to be investigated further as a central aspect of integrating machine learning and

368 other big-data approaches into water management and regulation.

369 A future scientific challenge remains to tie individual chemical dose-effects to multi-chemical

370 integrated hazard indices for human and environmental health; key to this is understanding how

371 multiple chemical exposures interact to produce cumulative health effects. Such efforts should

372 drive the determination of regulatory limits that machine learning frameworks, such as the one

373 described here, can be optimized towards.

\section{Conclusions:}

375 Contaminants in drinking water pose a significant threat to human health. With limited resources

376 and a growing number of emerging contaminants, direct testing of all possible contaminants is

377 infeasible. In this study, we develop a machine learning framework that can be used to prioritize

378 groundwater wells for testing using the limited resourced available. The machine learning

379 framework leverages diverse geospatial and chemical co-contaminant measurements to

380 accurately predict contaminants such as PFAS. Despite the high levels of missing data in the co-

381 contaminant data (in which only a small portion of the total possible measurements are available

382 for a given well), we demonstrate that there is sufficient structure to create accurate predictive

383 models. We found that co-contaminant features can be robustly predictive of unseen analytes, 
384 including PFAS, even when the machine learning model is trained on wells geographically

385 distinct from those it is applied to.

386 This result suggests our model for predicting PFAS has the potential to significantly accelerate

387 the ability to identify groundwater sources with concerningly high PFAS concentrations.

388 Strikingly, our model is projected to reduce the number of wells required to detect $70 \%$ of

389 concerningly high PFAS wells by a factor of five compared to a random sampling approach. In

390 general, we believe our approach could have broad utility in aiding regulatory agencies in

391 overseeing the management of anthropogenic contaminants in groundwater using predictive

392 analytics to guide testing strategies.

\section{Acknowledgements}

394 The authors thank Erica Kalve for offering support, encouragement, and expertise, as well as 395 colleagues at the San Francisco Bay Regional- and State of California- Water Resources Control

396 Boards for fruitful discussions of groundwater contamination and emerging contaminants; in 397 particular, the 2019 PFAS Summit and Datathon team. We would also like to thank Ari Frizzle for 398 moral support.

\section{Declaration of competing interest}

400 A.D. is a founder of and equity holder in Coral Genomics. The authors alone are responsible for 401 the views expressed in this publication and they do not necessarily represent the views, decisions 402 or policies of the institutions with which they are affiliated. 


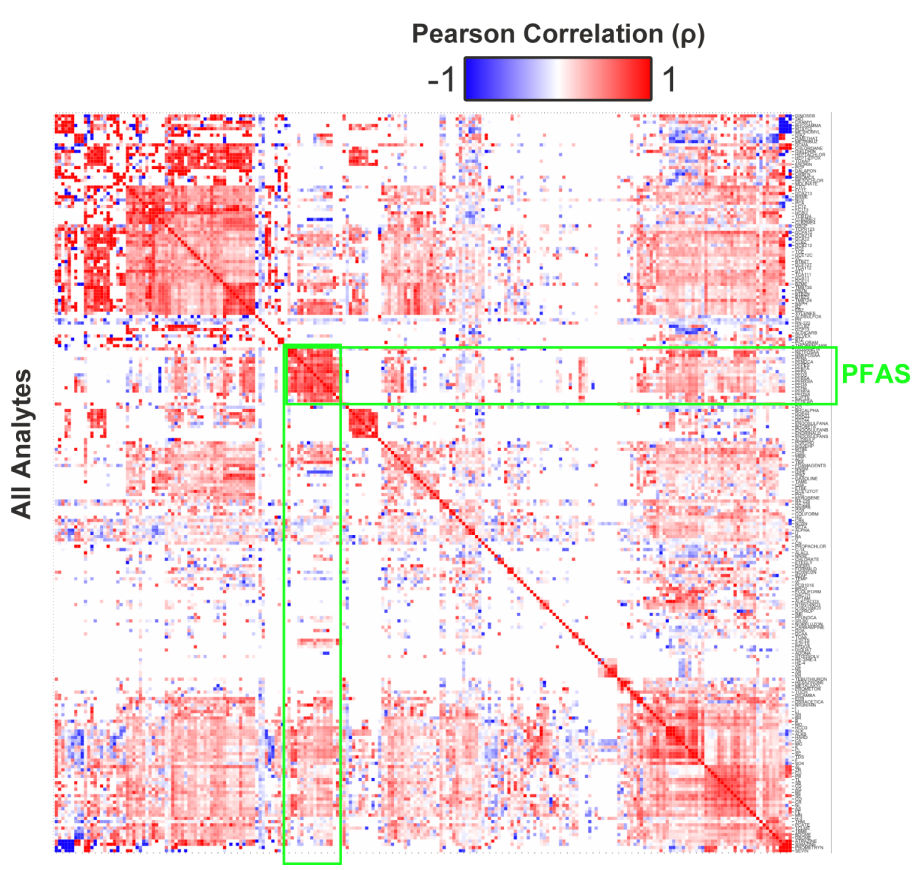

404 Figure S1. An overview of pairwise correlations between chemical and geospatial features in 405 the GAMA dataset.

406 A correlation matrix visualization of pairwise correlations between chemical and geospatial 407 features. Each cell represents the Pearson correlation between the respective elements as shown in 408 the yellow and purple scatter plots. The color scale ranges from red to blue, corresponding to a 409 correlation between 1.0 and -1.0. The PFAS set of compounds is highlighted in green (this region 410 overlaps with the data displayed in Figure 2C). 

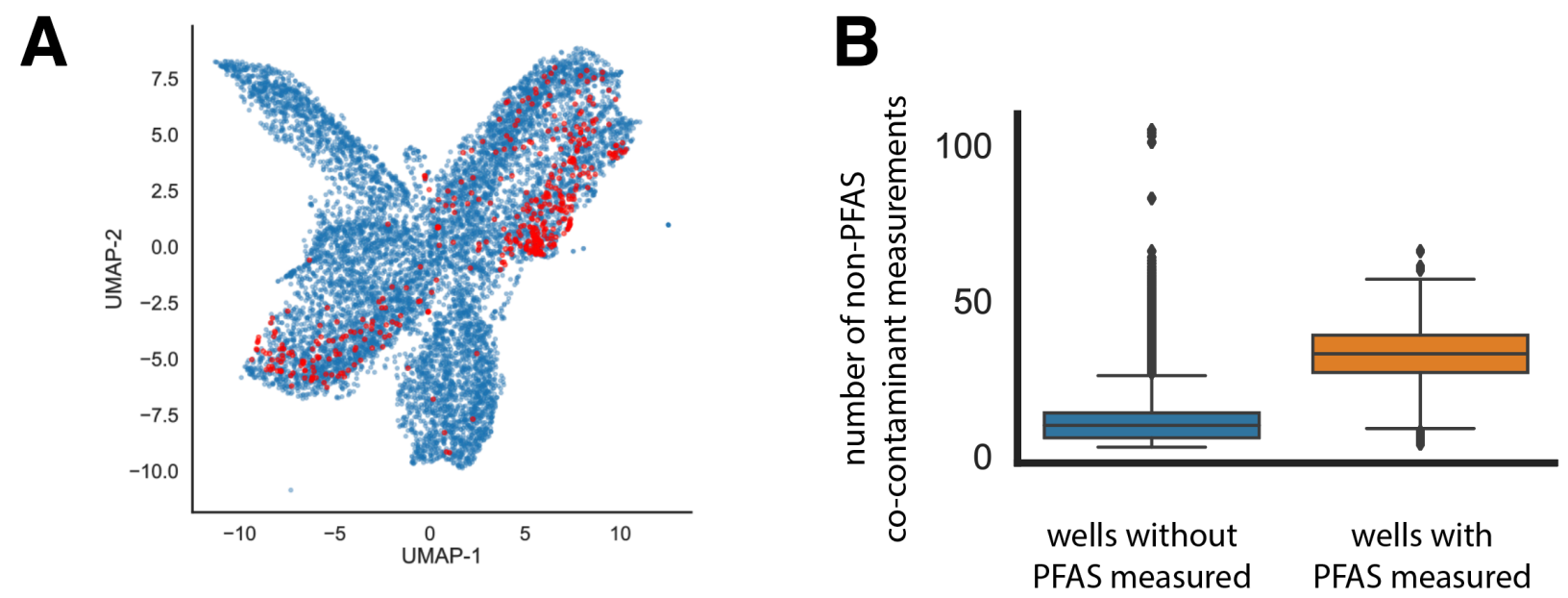

413 Figure S2. Comparison between wells that have had PFAS measured and those that have not

414 (a) After performing PCA on the co-contaminates dataset across all wells, dimensionality

415 reduction was performed using UMAP. Wells that have had PFAS measurements are highlighted

416 in red. (b) Boxplot showing the difference in the number of analytes measured in wells that have

417 had a PFAS measurement compared to those that have not had a PFAS measurement. Boxplots

418 denoting interquartile range, 1.5 times this distribution with whiskers, and outliers as dots. 


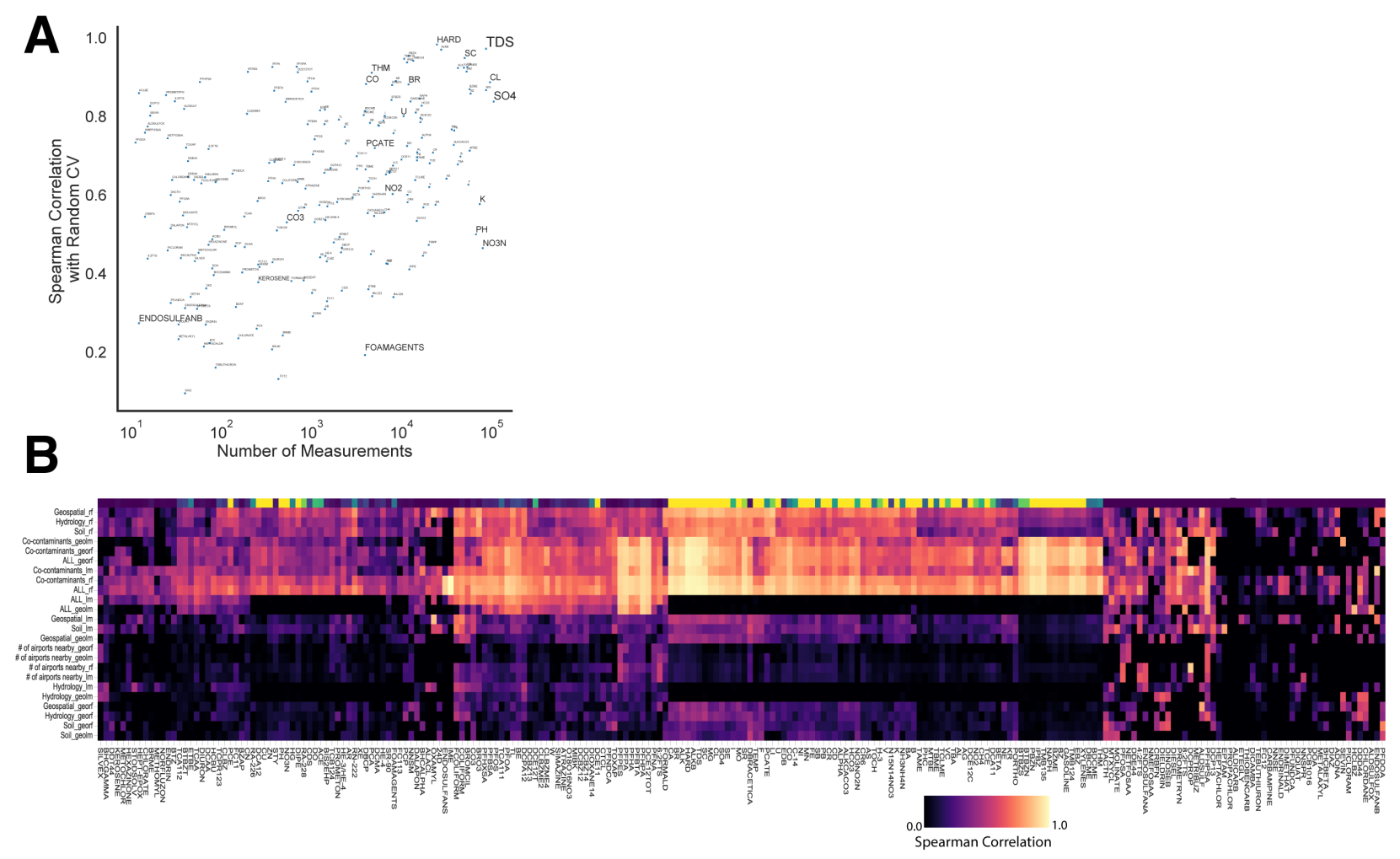

Figure S3. Results of application of model to all 228 chemicals.

422 (a) Scatter plot of the number of measurements on a $\log 10$ scale (x-axis) vs. the average Spearman

423 correlation of the test set performance across all 10 folds for a machine learning model trained to

424 predict that chemical analyte.

425 (b) Spearman correlation of the different model types (rows) applied to predicting each of the 228

426 chemical analytes (columns). Georf = geospatially clustered cross validation random forest. Geolm

427 = geospatially clustered linear model. $1 \mathrm{~m}=$ linear model. $\mathrm{rf}=$ random forest. First row is an a 428 colorimetric representation of the number of total measurements there were available for the 429 corresponding analyte in the dataset.

\section{References:}


433 Alimi, H., Ertel, T., Schug, B., 2003. Fingerprinting of hydrocarbon fuel contaminants:

434 Literature review. Environ. Forensics. https://doi.org/10.1080/15275920303489

435 Barrett, M.H., Hiscock, K.M., Pedley, S., Lerner, D.N., Tellam, J.H., French, M.J., 1999. Marker

436 species for identifying urban groundwater recharge sources: A review and case study in

437 Nottingham, UK. Water Res. https://doi.org/10.1016/S0043-1354(99)00021-4

438 Biege, L.B., Hurtt, M.E., Frame, S.R., O’Connor, J.C., Cook, J.C., 2001. Mechanisms of

439 extrahepatic tumor induction by peroxisome proliferators in male CD rats. Toxicol. Sci. 60,

$440 \quad 44-55$. https://doi.org/10.1093/toxsci/60.1.44

441 California Natural Resources Agency, n.d. Periodic Groundwater Level Measurements - Datasets

442 - California Natural Resources Agency Open Data [WWW Document]. URL

443 https://data.cnra.ca.gov/dataset/periodic-groundwater-level-measurements (accessed

$444 \quad 5.6 .21)$

445 California State Water Resources Control Board, 2020 GAMA Groundwater [WWW

446 Document]. Groundw. Ambient Monit. URL

447 https://gamagroundwater.waterboards.ca.gov/gama/gamamap/public/Default.asp (accessed

$448 \quad 4.10 .21)$.

449 Carle, D., 2015. Introduction to Water in California. University of California Press.

450 Conder, J.M., Hoke, R.A., De Wolf, W., Russell, M.H., Buck, R.C., 2008. Are PFCAs

451 bioaccumulative? A critical review and comparison with regulatory criteria and persistent

452 lipophilic compounds. Environ. Sci. Technol. https://doi.org/10.1021/es070895g 
453 Crone, B.C., Speth, T.F., Wahman, D.G., Smith, S.J., Abulikemu, G., Kleiner, E.J., Pressman, 454 J.G., 2019. Occurrence of per- and polyfluoroalkyl substances (PFAS) in source water and 455 their treatment in drinking water. Crit. Rev. Environ. Sci. Technol. 49, 2359-2396.

456 https://doi.org/10.1080/10643389.2019.1614848

457 De Silva, A.O., Armitage, J.M., Bruton, T.A., Dassuncao, C., Heiger-Bernays, W., Hu, X.C., 458 Kärrman, A., Kelly, B., Ng, C., Robuck, A., Sun, M., Webster, T.F., Sunderland, E.M., 2021. PFAS Exposure Pathways for Humans and Wildlife: A Synthesis of Current Knowledge and Key Gaps in Understanding. Environ. Toxicol. Chem.

Dodder, N.G., Maruya, K.A., Lee Ferguson, P., Grace, R., Klosterhaus, S., La Guardia, M.J., https://doi.org/10.1002/etc.4935 Lauenstein, G.G., Ramirez, J., 2014. Occurrence of contaminants of emerging concern in

Domingo, J.L., Nadal, M., 2019. Human exposure to per- and polyfluoroalkyl substances (PFAS) mussels (Mytilus spp.) along the California coast and the influence of land use, storm water

discharge, and treated wastewater effluent. Mar. Pollut. Bull. 81, 340-346. https://doi.org/10.1016/j.marpolbul.2013.06.041

470 Douglas, G.S., Emsbo-Mattingly, S.D., Stout, S.A., Uhler, A.D., McCarthy, K.J., 2007.

471 Chemical fingerprinting methods, in: Introduction to Environmental Forensics. Elsevier Inc., pp. 311-454. https://doi.org/10.1016/B978-012369522-2/50010-5

473 Elevation Point Query Service [WWW Document], n.d. URL https://nationalmap.gov/epqs/ 
475 Ericson, I., Nadal, M., Van Bavel, B., Lindström, G., Domingo, J.L., 2008. Levels of 476 perfluorochemicals in water samples from Catalonia, Spain: Is drinking water a significant 477 contribution to human exposure?, in: Environmental Science and Pollution Research. Springer, pp. 614-619. https://doi.org/10.1007/s11356-008-0040-1

Fasano, W.J., Carpenter, S.C., Gannon, S.A., Snow, T.A., Stadler, J.C., Kennedy, G.L., Buck, R.C., Korzeniowski, S.H., Hinderliter, P.M., Kemper, R.A., 2006. Absorption, distribution,

Fischer, G., Nachtergaele, F., Prieler, S., van Velthuizen, H.., Verelst, L., Wiberg, D., 2008. metabolism, and elimination of 8-2 fluorotelomer alcohol in the rat. Toxicol. Sci. 91, 341-

Frömel, T., Knepper, T.P., 2010. Biodegradation of fluorinated alkyl substances. Rev. Environ. Contam. Toxicol. 208, 161-177. https://doi.org/10.1007/978-1-4419-6880-7_3

Giesy, J.P., Kannan, K., 2001. Global distribution of perfluorooctane sulfonate in wildlife. Environ. Sci. Technol. 35, 1339-1342. https://doi.org/10.1021/es001834k Derived Per- and Polyfluoroalkyl Substances in a Wastewater Treatment Plant. Environ. Sci. Technol. 52, 13212-13221. https://doi.org/10.1021/acs.est.8b04028 
precursors in AFFF-impacted groundwater and soil. Environ. Sci. Technol. 47, 8187-8195. https://doi.org/10.1021/es4018877

496

497

498

Houtz, E.F., Sedlak, D.L., 2012. Oxidative conversion as a means of detecting precursors to perfluoroalkyl acids in urban runoff. Environ. Sci. Technol. 46, 9342-9349. https://doi.org/10.1021/es302274g

Hu, X.C., Andrews, D.Q., Lindstrom, A.B., Bruton, T.A., Schaider, L.A., Grandjean, P., Lohmann, R., Carignan, C.C., Blum, A., Balan, S.A., Higgins, C.P., Sunderland, E.M., 2016. Detection of Poly- and Perfluoroalkyl Substances (PFASs) in U.S. Drinking Water Linked to Industrial Sites, Military Fire Training Areas, and Wastewater Treatment Plants. Environ. Sci. Technol. Lett. 3, 344-350. https://doi.org/10.1021/acs.estlett.6b00260

Hurley, S., Houtz, E., Goldberg, D., Wang, M., Park, J.S., Nelson, D.O., Reynolds, P., Bernstein, L., Anton-Culver, H., Horn-Ross, P., Petreas, M., 2016. Preliminary associations between the detection of perfluoroalkyl acids (PFAAs) in drinking water and serum concentrations in a sample of California women. Environ. Sci. Technol. Lett. 3, 264-269. https://doi.org/10.1021/acs.estlett.6b00154

Kannan, K., Corsolini, S., Falandysz, J., Fillmann, G., Kumar, K.S., Loganathan, B.G., Mohd, M.A., Olivero, J., Van Wouwe, N., Yang, J.H., Aldous, K.M., 2004. Perfluorooctanesulfonate and related fluorochemicals in human blood from several countries. Environ. Sci. Technol. 38, 4489-4495. https://doi.org/10.1021/es0493446

Kibbey, T.C.G., Jabrzemski, R., O’Carroll, D.M., 2020. Supervised machine learning for source allocation of per- and polyfluoroalkyl substances (PFAS) in environmental samples. 
516 Kim, K., Bennett, D.H., Calafat, A.M., Hertz-Picciotto, I., Shin, H.M., 2020. Temporal trends 517 and determinants of serum concentrations of per- and polyfluoroalkyl substances among 518 Northern California mothers with a young child, 2009-2016. Environ. Res. 186, 109491. 519 https://doi.org/10.1016/j.envres.2020.109491

Le, S.T., Kibbey, T.C.G., Weber, K.P., Glamore, W.C., O’Carroll, D.M., 2021. A groupcontribution model for predicting the physicochemical behavior of PFAS components for understanding environmental fate. Sci. Total Environ. 764, 142882. https://doi.org/10.1016/j.scitotenv.2020.142882

Lewis, R.C., Johns, L.E., Meeker, J.D., 2015. Serum biomarkers of exposure to perfluoroalkyl substances in relation to serum testosterone and measures of thyroid function among adults and adolescents from NHANES 2011-2012. Int. J. Environ. Res. Public Health 12, 60986114. https://doi.org/10.3390/ijerph120606098

Maantay, J., 2002. Zoning law, health, and environmental justice: What's the connection?, in: Journal of Law, Medicine and Ethics. Blackwell Publishing Inc., pp. 572-593. https://doi.org/10.1111/j.1748-720X.2002.tb00427.x

531 Martin, J.W., Ellis, D.A., Mabury, S.A., Hurley, M.D., Wallington, T.J., 2006. Atmospheric 532 Chemistry of Perfluoroalkanesulfonamides: Kinetic and Product Studies of the OH Radical and $\mathrm{Cl}$ Atom Initiated Oxidation of $N$-Ethyl Perfluorobutanesulfonamide. Environ. Sci. Technol. 40, 864-872. https://doi.org/10.1021/es051362f 
fluorotelomer alcohols in isolated rat hepatocytes. Chem. Biol. Interact. 155, 165-180. https://doi.org/10.1016/j.cbi.2005.06.007

Mohai, P., Pellow, D., Roberts, J.T., 2009. Environmental Justice. Annu. Rev. Environ. Resour. 34, 405-430. https://doi.org/10.1146/annurev-environ-082508-094348

Mohai, P., Saha, R., 2015. Which came first, people or pollution? A review of theory and evidence from longitudinal environmental justice studies. Environ. Res. Lett. 10, 125011. https://doi.org/10.1088/1748-9326/10/12/125011

Parsons, J.R., Sáez, M., Dolfing, J., de Voogt, P., 2008. Biodegradation of perfluorinated

Plumlee, M.H., Kennedy/Jenks Consultants, Gurr, C.J., CDM Smith, Reinhard, M., 2011. compounds. Rev. Environ. Contam. Toxicol. 196, 53-71. https://doi.org/10.1007/978-0387-78444-1_2

Plumlee, M.H., Larabee, J., Reinhard, M., 2008. Perfluorochemicals in water reuse. Attenuation of Emerging Contaminants in Streams Augmented with Recycled Water I WateReuse Association.

551 Prevedouros, K., Cousins, I.T., Buck, R.C., Korzeniowski, S.H., 2006. Sources, fate and 552 Chemosphere 72, 1541-1547. https://doi.org/10.1016/j.chemosphere.2008.04.057

554 Rayne, S., Forest, K., 2009. Perfluoroalkyl sulfonic and carboxylic acids: A critical review of 555 physicochemical properties, levels and patterns in waters and wastewaters, and treatment 
methods. J. Environ. Sci. Heal. - Part A Toxic/Hazardous Subst. Environ. Eng. https://doi.org/10.1080/10934520903139811

Sengupta, A., Lyons, J.M., Smith, D.J., Drewes, J.E., Snyder, S.A., Heil, A., Maruya, K.A., 2014. The occurrence and fate of chemicals of emerging concern in coastal urban rivers receiving discharge of treated municipal wastewater effluent. Environ. Toxicol. Chem. 33, 350-358. https://doi.org/10.1002/etc.2457

Sjogren, P., Montse, R., Lampa, E., Salihovic, S., van Bavel, B., Lind, L., Lind, P.M., 2016. Circulating levels of perfluoroalkyl substances are associated with dietary patterns - A cross sectional study in elderly Swedish men and women. Environ. Res. 150, 59-65. https://doi.org/10.1016/j.envres.2016.05.016

State Water Resources Control Board, 2020. California State Water Resources Control Board.

US EPA, O., 2015. Occurrence Data for the Unregulated Contaminant Monitoring Rule.

Vestergren, R., Cousins, I.T., 2009. Tracking the pathways of human exposure to perfluorocarboxylates. Environ. Sci. Technol. https://doi.org/10.1021/es900228k Comprehensive Global Emission Inventory of C4-C10 Perfluoroalkanesulfonic Acids (PFSAs) and Related Precursors: Focus on the Life Cycle of C8-Based Products and Ongoing Industrial Transition. Environ. Sci. Technol. 51, 4482-4493. 
577 Xu, Y., Jurkovic-Mlakar, S., Li, Y., Wahlberg, K., Scott, K., Pineda, D., Lindh, C.H., Jakobsson,

578 K., Engström, K., 2020. Association between serum concentrations of perfluoroalkyl

579 substances (PFAS) and expression of serum microRNAs in a cohort highly exposed to

$580 \quad$ PFAS from drinking water. Environ. Int. 136, 105446.

$581 \quad$ https://doi.org/10.1016/j.envint.2019.105446

582 Young, C.J., Mabury, S.A., 2010. Atmospheric perfluorinated acid precursors: Chemistry, 583 occurrence, and impacts. Rev. Environ. Contam. Toxicol. 208, 1-109.

$584 \quad$ https://doi.org/10.1007/978-1-4419-6880-7_1

585 Zhang, Y., Schaap, M.G., Zha, Y., 2018. A High-Resolution Global Map of Soil Hydraulic 586 Properties Produced by a Hierarchical Parameterization of a Physically Based Water

587 Retention Model. Water Resour. Res. 54, 9774-9790.

$588 \quad$ https://doi.org/10.1029/2018WR023539 